\title{
Non-surgical orthodontic adult molar crossbite correction and sleep apnea
}

\begin{abstract}
Introduction: Diagnosis and treatment planning for a bilateral molar crossbite of an adult patient involves understanding its etiology, quantifying the discrepancy while considering the potential for surgical maxillary expansion due to the limitations of conventional orthodontic systems. This case report illustrates a highly successfully and novel nonsurgical, non-extraction approach to correction of an adult bilateral molar crossbite with new orthodontics mechanics. It also provides the adjunctive effect of alleviating a constricted upper airway potentially benefitting patients with sleep apnea.
\end{abstract}

Case report: The patient, who is a 24 -year-old female with a bilateral molar crossbite and transverse discrepancy of $3 \mathrm{~mm}$ associated with an anterior open bite, successfully completes treatment with a maxillary expansion appliance immediately followed by nonsurgical, non-extraction orthodontic treatment in a little over 12 months. Post treatment clinical and radiographic results show dramatic esthetic and functional improvement with the elimination of a bilateral molar crossbite and the establishment of a stable occlusion with good intercuspation. In addition, the post treatment cephalogram shows radiographic evidence of an increased upper airway dimension.

Conclusion: This case report demonstrates the potential of using a maxillary expansion appliance with non-surgical, non-extraction orthodontic therapy for an adult bilateral molar crossbite followed by the use of a system of braces that immediately moves the tooth root(s) to their final position with alveolar bone remodeling and short treatment time. In addition, it illustrates the possible adjunctive benefit of increasing the patient's airway which could provide relief for the sleep apnea patient.
Volume 5 Issue 5 - 2016

\author{
Anthony DViazis, ' Evangelos Viazis, ${ }^{2}$ Tom C \\ Pagonis ${ }^{3}$ \\ 'Orthodontist, Private Practice, Dallas, Texas, U.S.A \\ ${ }^{2}$ Private Practice, Athens, Greece \\ ${ }^{3}$ Assistant Clinical Professor (former), Harvard School of Dental \\ Medicine, Boston, MA, U.S.A
}

Correspondence: Tom C Pagonis, Assistant Clinical Professo (former), Harvard School of Dental Medicine, Boston, MA, U.S.A, Tel I 617-432-5846, Fax 1617-432-090।,

Email tom_pagonis@hsdm.harvard.edu

Received: November 25, 2016 | Published: December 0I, 2016

\section{Introduction}

The clinical manifestation of a molar crossbite and its correction is complex requiring proper diagnosis and the development of an appropriate treatment plan. A posterior crossbite is defined as any abnormal buccal-lingual relation of opposing posterior teeth creating inadequate transversal relations such that buccal cusps of posterior maxillary teeth occlude with the central fossae of opposing mandibular teeth. ${ }^{1}$ In effect, when compared to normal, the buccal-lingual relationships are reversed in a posterior crossbite. The incidence of this malocclusion varies among the Hispanic, African American and Caucasian populations at $7.3 \%, 9.6 \%$ and $9.1 \%$, respectively. ${ }^{2,3}$ The etiology of this malocclusion is typically multifactorial and can include a combination of dental, skeletal and functional components. ${ }^{4}$ Specific disease entities which can contribute to this clinical presentation include Treacher Collins, Marfan Syndrome, Kippel-Fell Syndrome, Duchenne Muscular Dystrophy in addition to craniosynostosis associated with Crouzon's and Apert's Disease. Functional habits such as thumb sucking and sleep apnea are also responsible for constricted maxillary arches. ${ }^{4,5}$ Of particular interest for a sleep apnea patient with a clinical presentation of a narrow maxilla and associated bilateral molar crossbite is the possible adjunctive alleviation of a constricted airway after maxillary expansion and orthodontic treatment. Since a bilateral posterior crossbite does not exhibit spontaneous correction, rapid maxillary expansion, also known as palatal expansion, should be attempted at the start of orthodontic treatment particularly for a patient 18 years old or younger. ${ }^{6,7}$ Adult patients seeking treatment could be subjected to surgical correction of their crossbite. This case report illustrates the utilization of a maxillary expansion appliance in an adult patient non-surgically with advances in mechanotherapy and diagnosis which now allow the clinician to treatment plan certain skeletal cases with non-extraction, non-surgical orthodontic treatment in short treatment times. ${ }^{89}$ It also illustrates the possible adjunctive benefit of relieving a patient's constricted airway which could provide relief for the sleep apnea patient.

\section{Case report}

\section{Diagnosis}

The patient is a 24-year-old female who presented to the second author's private practice in Athens, Greece with a chief complaint of esthetic concerns with her smile and with difficulty chewing (Figure 1). On examination the patient has a mesoproscopic facial form, with an open bite of $5 \mathrm{~mm}$, measured from maxillary and mandibular incisal edges and an overjet of $4 \mathrm{~mm}$. The maxillary arch is relatively narrow when compared to the mandible with a molar transverse discrepancy of $3 \mathrm{~mm}$. Functionally, the patient only occludes on her second premolars and molars while in maximum intercuspation. Although this patient did not exhibit sleep apnea symptoms, it is often associated with patients having a similar clinical presentation which may develop because of a constricted airway. ${ }^{4,5}$ Examination of the oral soft tissue, periodontium and dentition revealed slight marginal edema. 

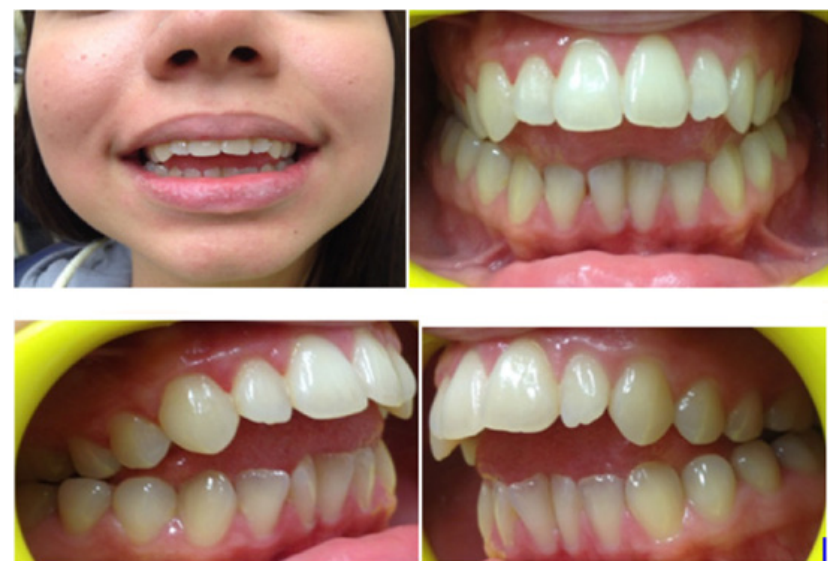

Figure IA Pre-treatment facial and intra-oral frontal view photographs.
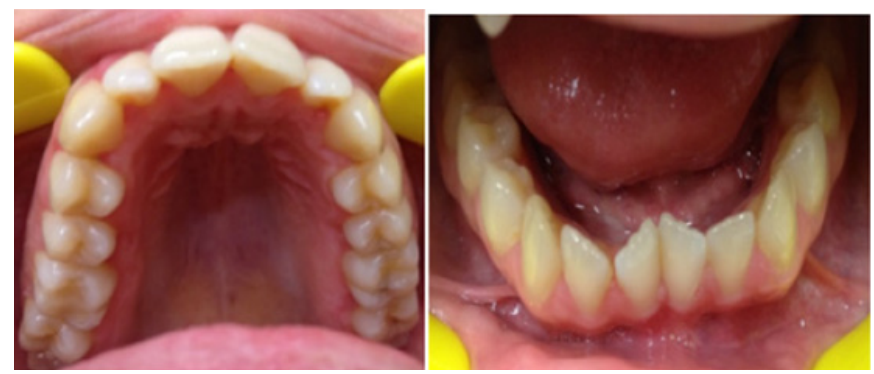

Figure I B Pre-treatment intra-oral occlusal view photographs.

\section{Treatment objectives}

Upon completion of clinical examination and review of pretreatment panoramic and lateral cephalogram radiographs the patient was treatment planned with a two phase approach which included a maxillary expansion appliance (phase 1) followed by orthodontic treatment (phase 2) and advised of the potentially favorable prognosis of a new non-extraction orthodontic treatment. She decided to pursue attempted maxillary expansion followed by non-surgical, non-extraction orthodontic treatment in order to correct her bilateral molar crossbite, obtain proper overjet and overbite relations, level and align her occlusion and restore satisfactory esthetics by utilizing the bracket technology system of Fastbraces ${ }^{\circledR}$ (Figure 2).
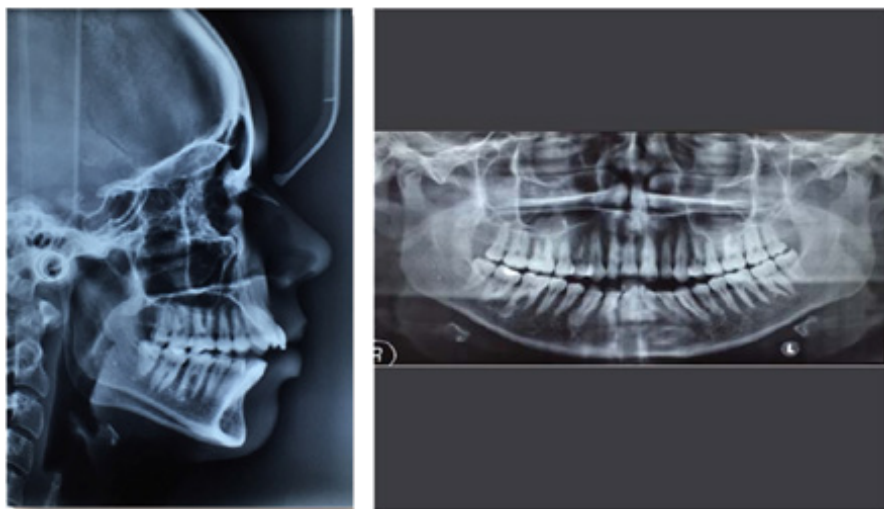

Figure 2 Pre-treatment lateral cephalogram and panoramic radiographs.

\section{Treatment progress}

At the initial treatment appointment, the patient was fitted with a Hyrax maxillary expansion appliance and was seen on a monthly basis. She wore her expander for a total of four months with one adjustment that took place at the two-month appointment. Also at two months, brackets were placed on the mandibular anterior teeth with subsequent application of brackets on remaining mandibular teeth and all maxillary teeth after the completion of phase 1 or maxillary expansion appliance treatment. Upon completion of orthodontic treatment and removal of all braces, a bonded maxillary fixed retainer was placed lingually from canine to canine. Total treatment time including the use of a maxillary expansion appliance took a little over 12 months with appointments scheduled approximately on a monthly basis with no interproximal reduction of mandibular teeth in proximal contact.

\section{Treatment results}

Clinical results along with photographs and radiographs comparing pre and post treatment show dramatic esthetic and functional improvement, elimination of the bilateral posterior crossbite and correction of anterior guidance with a stable occlusion. Overjet and overbite was measured at between 1 to $2 \mathrm{~mm}$ with a treatment time of a little over 12 months (Figures $3 \& 4$ ). In addition, comparing pre with post treatment cephalograms shows radiographic evidence of an increased upper airway dimension.
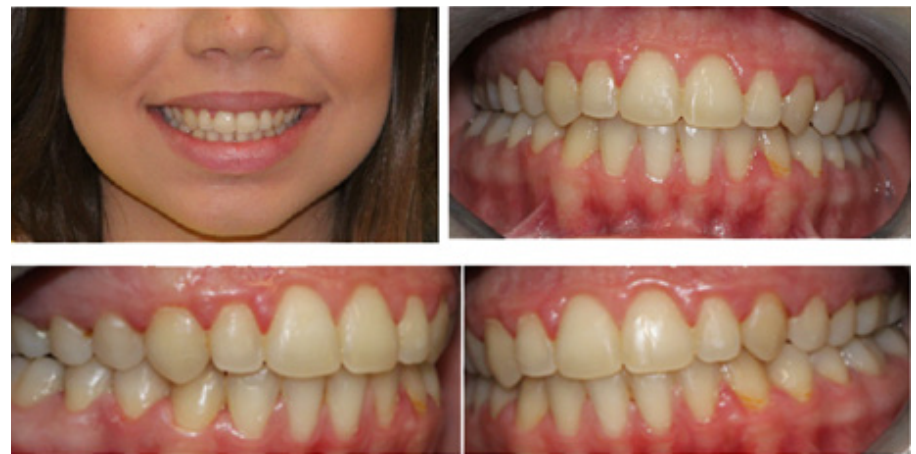

Figure 3A Post-treatment facial and intra-oral frontal view photographs.
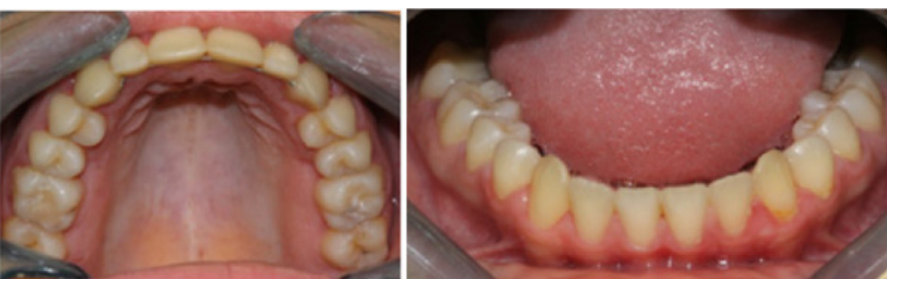

Figure 3B Post-treatment intra-oral occlusal view photographs.
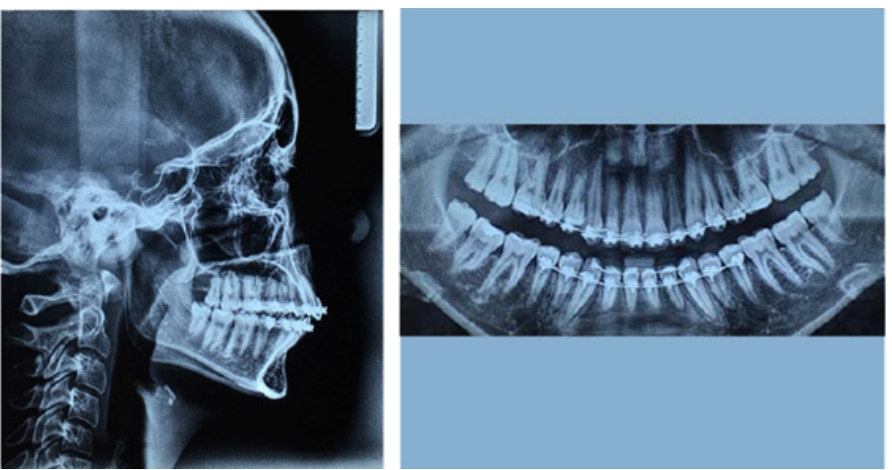

Figure 4 Post-treatment lateral cephalogram and panoramic radiographs. 


\section{Discussion}

The ultimate goal in treating skeletal malocclusions associated with both bilateral posterior crossbites with an associated anterior open bite is to create dentoalveolar changes that correct this imbalance. The strategy for selecting a two phased approach for treatment of a bilateral posterior crossbite which includes both the utilization of a maxillary expansion appliance followed by orthodontic treatment is limited by

a. The age of the patient as it relates to the potential of maxillary expansion due to ossification of the midpalatal suture.

b. The limitations of conventional orthodontic treatment. The literature continues to suggest that when untreated, crossbites can lead to long term and permanent growth alteration thereby necessitating early treatment intervention. ${ }^{10}$

Yet little evidence exists on using a similar treatment protocol for an adult patient. This case report demonstrates the potential of utilizing a maxillary expansion appliance for a 24-year-old adult patient for the first phase of treatment to correct gross bilateral molar crossbite by initially tipping maxillary posterior teeth buccally rather than expanding the maxilla at the midpalatal suture thereby markedly reducing the transverse discrepancy. Non-surgical adult expansion can now be done on cases of end on to slightly lingual (i.e. 1 to 3 $\mathrm{mm}$ ) bilateral posterior molar crossbite. Severe cases (i.e. $3 \mathrm{~mm}$ or more of maxillary molar lingual crossbite) especially when the maxillary molar is one half way or more lingual to the mandibular molar may require maxillary jaw surgery. The maxillar y expander is turned every other day $(.25 \mathrm{~mm}$ or one turn) and is removed when the maxillary molar occlusal surface is half way buccal to the mandibular molar surface but not in complete buccal crossbite. The same day the maxillary expander is removed, full maxillary braces and the wire are placed and the elastics are initiated on a full time basis. The mandibular braces would have been placed at a prior patient visit. The torque applied by the Fastbraces ${ }^{\circledR}$ technologies square wire bracket systems immediately begins up righting the roots of the tipped molars into their final upright position. This would not be possible with old style braces that use a round wire which by definition applies no torque. The subsequent application of the orthodontic system Fastbraces, ${ }^{\circledR}$ a new technology system of braces that utilizes the application of torque which facilitates root up righting and thus alveolar bone remodeling and development thereby correcting transverse discrepancies while, in this particular case, also correcting an associated anterior open bite in short treatment times. There is a growing body of literature and accompanying interest in upper airway shape and dimensions primarily due to the relationship between upper airway configuration and sleep-disordered breathing including obstructive sleep apnea. ${ }^{11,12}$ Although several modalities such as computed tomography scanning and magnetic resonance imaging are available, the lateral cephalogram remains an important, readily available and less expensive radiographic screening tool for obstructive sleep apnea. ${ }^{13}$ Analysis and upper airway measurement of landmarks on cephalograms which compare sleep apnea and healthy patients show a clear tendency for sleep apnea patients to have smaller airway dimensions. ${ }^{14,15}$ Although this patient did not demonstrate or present with symptoms of sleep apnea, comparative pre and post treatment cephalograms suggest a larger upper airway opening at the approximate areas between the dorsum of the tongue and posterior pharyngeal wall. While additional clinical research is necessary this treatment presents a possible adjunctive benefit to the sleep apnea patient with a constricted upper airway. This case illustrates the dramatic non-surgical correction of a bilateral molar crossbite with the Fastbraces ${ }^{\circledR}$ system with possible additional benefits of improving upper airway dimensions. It remains a valuable adjunctive system to comprehensive dental treatment planning of a complex adult case.

\section{Conclusion}

This case report demonstrates the successful non-extraction, non-surgical outcome and correction of an adult bilateral molar crossbite accompanied by an anterior open bite with Fastbraces ${ }^{\circledR}$, a new technology system of braces that facilitates the continuation of eruption while inducing alveolar bone remodeling and development in short treatment times. ${ }^{16}$ This treatment offers consideration as a possible adjunct to patients also presenting with sleep apnea by improving upper airway dimensions. Carefully diagnosed skeletal malocclusions of this magnitude for an adult patient that are typically treatment planned for maxillary jaw surgery can potentially be treated orthodontically without extractions in a timely manner.

\section{Acknowledgments}

None.

\section{Conflicts of interest}

The authors declare that there are no conflicts of interest.

\section{Funding details}

None.

\section{References}

1. O'Donnell S, Nanda RS, Gosh J. Perioral forces and dental changes resulting from mandibular lip bumper treatment. Am J Orthod Dent Orthop. 1998;113(3):247-255.

2. Silva Filho OG, Montes LAP, Torelly LF. Rapid maxillary expansion in the dentition evaluated through posteranterior cephalometric analysis. Am J Orthod Dentofacial Orthop. 1995;107(3):268-275.

3. Bartzela T, Jonas I. Long-term stability of unilateral posterior crossbite correction. Angle Orthod. 2007;77(2):237-243.

4. Allen D, Rebellato J, Sheats R, et al. Skeletal and dental contributions to posterior crossbites. Angle Orthodontist. 2003;73(5):515-524.

5. Ennedy DB, Osepchook M. Unilateral Posterior Crossbite with Mandibular Shift:A Review. J Can Dent Assoc. 2005;71(8):569-573.

6. Mc Namara JA. Early intervention in the transverse dimension:is it worth the effort? Am J Orthod Dent Orthop. 2002;121(6):572-574.

7. Vogel CJ. An interview with James A. McNamara Jr. Dental Press Journal of Orthodontics. 2011;16(3):32-53.

8. Viazis AD, Viazis E, Pagonis TC. The Fallacy of Tongue Thrust and NonSurgical Treatment of a Severe Anterior Open Bite. J Dent Health Oral Disord Ther. 2016;4(4):00120.

9. Viazis AD, Viazis E, Pagonis TC. Non-Surgical Orthodontic Treatment of an Orthognathic Surgical Case. J Dent Health Oral Disord Ther. 2016;4(5):00128.

10. Pinto AS, Buschang PH, Throckmorton GS, et al. Morphological and positional asymmetries of young children with functional unilateral posterior crossbite. Am J Orthod Dentofacial Orthop. 2001;120(5):513-520. 
11. Katyal V, Pamula Y, Martin AJ, et al. Craniofacial and upper airway morphology in pediatric sleep- disordered breathing:systematic review and meta-analysis. American Journal of Orthodontics and Dentofacial Orthopedics. 2013;143(1):20-30.

12. Flores Mir C, Korayem M, Heo G, et al. Craniofacial morphological characteristics in children with obstructive sleep apnea syndrome:a systematic review and meta-analy-sis. Journal of the American Dental Association. 2013;144:269-277.

13. Vizzotto MB, Liedke GS, Delamare EL, et al. A comparative study of lateral cephalograms and cone-beam computed tomographic images in upper airway assessment. Eur J Orthod. 2012;34(3):390-393.
14. Kim J, In K, You S, et al. Prevalence of sleep-disordered breathing in middle-aged Korean men and women. Am J Respir Crit Med. 2004;70(10):1108-1113.

15. Young T, Palta M, Dempsey J, et al. The occurrence of sleepdisordered breathing among middle-aged adults. $N$ Engl $\mathrm{J}$ Med. 1993;328(17):1230-1235.

16. Viazis AD, Viazis E, Pagonis TC. The Concept of a New Dental Disease:Orthodontosis and Orthodontitis. J Dent Health Oral Disord Ther. 2014;1(5):00030. 